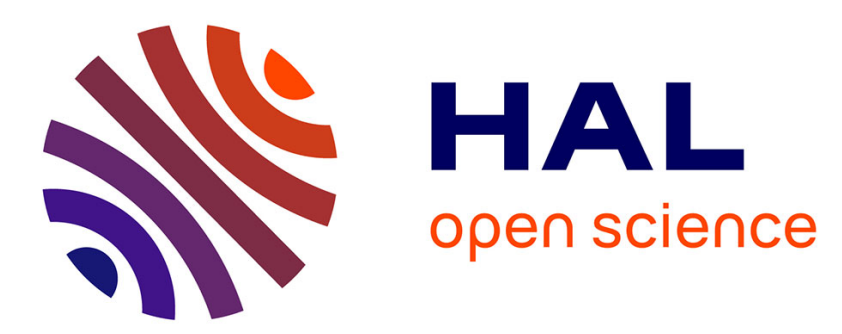

\title{
TOF-SIMS Imaging of Biological Tissue Sections and Structural Determination Using Tandem MS
}

\author{
Sebastiaan van Nuffel, Alain Brunelle
}

\section{To cite this version:}

Sebastiaan van Nuffel, Alain Brunelle. TOF-SIMS Imaging of Biological Tissue Sections and Structural Determination Using Tandem MS. Mass Spectrometry Imaging of Small Molecules, 2437, Springer US, pp.77-86, 2021, Methods in Molecular Biology, 10.1007/978-1-0716-2030-4_5 . hal-03479024

\section{HAL Id: hal-03479024 https://hal.science/hal-03479024}

Submitted on 14 Dec 2021

HAL is a multi-disciplinary open access archive for the deposit and dissemination of scientific research documents, whether they are published or not. The documents may come from teaching and research institutions in France or abroad, or from public or private research centers.
L'archive ouverte pluridisciplinaire HAL, est destinée au dépôt et à la diffusion de documents scientifiques de niveau recherche, publiés ou non, émanant des établissements d'enseignement et de recherche français ou étrangers, des laboratoires publics ou privés. 
TOF-SIMS Imaging of Biological Tissue Sections and Structural Determination using Tandem MS

Sebastiaan Van Nuffel ${ }^{1,2}$ \& Alain Brunelle ${ }^{3}$

1 Faculty of Science and Engineering, Maastricht University, 6229EN Maastricht, The Netherlands.

2 Maastricht MultiModal Molecular Imaging Institute (M4i), Maastricht University, 6229ER Maastricht, The Netherlands.

3 Sorbonne Université, CNRS, Laboratoire d'Archéologie Moléculaire et Structurale, LAMS, 4 place Jussieu, 75005 Paris, France.

\section{Abstract}

Over the past couple of years, imaging mass spectrometry (IMS) has arisen as a powerful tool to answer research questions in the biomedical field. Imaging mass spectrometry allows for label-free chemical imaging by providing full molecular information. The IMS technique best positioned for cell and tissue analysis is time-of-flight secondary ion mass spectrometry (ToF-SIMS) because it has the best spatial resolution of all the molecular IMS techniques and can detect many biochemical species and especially lipids with high sensitivity. Because one must rely on the mass and isotopic pattern of an ion in combination with positive correlations with lower mass fragments to help identify its structure, one major problem during ToF-SIMS experiments is the ambiguity when assigning a molecule to a certain mass peak. The solution are instruments with tandem MS capabilities as was already the case for many MALDI-ToF instruments more than a decade ago. It's been a few years since instruments with this capability were introduced and a number of interesting publications have been produced highlighting the advantages in biological SIMS work. Here, we present a protocol describing how tandem MS can be used to elucidate the structure of unknown or ambiguous mass peaks in biological tissue samples observed during ToF-SIMS imaging based on our experiences. 
Key words

SIMS, Time-of-flight, Orbitrap, Tandem MS, Imaging mass spectrometry, Lipids, Fatty acids

Running head

TOF-SIMS imaging and Tandem-MS of tissue sections

Introduction

There are a variety of articles and books, which explain the basics of ToF-SIMS imaging, and we refer the reader to those for a more profound understanding [1-3]. However, we will give a brief summary for the sake of clarity. Simply put, secondary ion mass spectrometry (SIMS) refers to the use of primary ions, e.g. $\mathrm{Bi}_{3}{ }^{+}$ions produced by a Bi liquid metal ion gun (LMIG), to generate secondary ions from solid samples. Firing primary ions at the sample causes a collision cascade that causes molecules from the top 1-3 monolayers of material at the sample surface to desorb and ionize. Typically, these ions are then sent to a mass analyzer using a static electric field and their mass-to-charge $(\mathrm{m} / \mathrm{z})$ ratio is determined via a timeof-flight (ToF) measurement. Because the primary ions can be focused onto the sample as a narrow beam, it is possible to raster the beam across the sample to generate a hyperspectral $2 \mathrm{D}$ image where every pixel is a full mass spectrum. One of the advantages of an imaging mass spectrometry technique such as ToFSIMS is the fact that the imaging is label-free, because individual molecules can be identified by characteristic mass peaks that show up in the ToF-SIMS mass spectrum. Using ToF-SIMS imaging, it is possible to visualize the spatial distribution of small molecules $(<2000 \mathrm{Da})$ in $2 \mathrm{D}$ with a spatial resolution comparable to that of a light microscope. If equipped with a sputter gun such as an $\mathrm{Ar}$ gas cluster ion beam (GCIB), it is also possible to acquire 3D image stacks with $<10 \mathrm{~nm}$ depth resolution by removing material through sputtering in between 2D ToF-SIMS imaging experiments. Because of these molecular imaging abilities, ToF-SIMS is extensively used for characterization in materials science and increasingly the life sciences. 
SIMS can also provide structural information, because SIMS generally produces both molecular ions and fragment ions. This fragmentation pattern allows one to determine the structure of unknown compounds. However, a sample can contain many unknown chemistries and the secondary ions of the different compounds present in the sample are all formed together after the impact of the primary ion, which is why a 'panoramic' detector with high transmission such as a ToF mass analyzer is typically used for SIMS. This property allows for label-free detection, but is a double-edged sword because it also means that a typical ToF-SIMS mass spectrum can be considered a summation of the spectra of the individual compounds present. In the case of ToF-SIMS imaging, it is possible to determine which mass peaks are positively correlated using principal component analysis or some other multivariate analysis technique. However, spatial colocation does not necessarily mean that these mass peaks all originate from one compound, particularly in the case of complex systems such as biological ones. This is further complicated by the fact that different compounds can produce similar fragmentation patterns with many of the same fragment ions. This is especially a problem when investigating biological materials, because biomolecules are often composed of the same building blocks. In addition, the mass resolution attainable with a ToF mass analyzer is typically limited to 10,000 , which means not all peaks are necessarily resolved. One mass peak can thus harbor multiple ions. All this obfuscates which mass peaks belong together, i.e. originate from the same compound. Finally, the secondary ion intensity and fragments produced using desorptionionization techniques such as ToF-SIMS are highly dependent on the chemical environment of the compounds. This so-called 'matrix effect' has made it very difficult to fingerprint and library approaches have proven largely ineffectual for ToF-SIMS. Even in simple systems, it is often necessary to work with reference materials in order to be certain of the identity of a mass peak of interest.

For these reasons, the manufacturers of ToF-SIMS instruments have come up with different strategies to implement MS/MS capabilities, as was already the case for many MALDI-ToF instruments more than a decade ago. MS/MS or Tandem MS is a technique where the ions of a particular $\mathrm{m} / \mathrm{z}$-ratio are selected 
and are then made to undergo fragmentation (usually through collision-induced dissociation or CID) before being mass analyzed again. While the first mass spectrum (MS1) is a summation of the molecular and fragment ions generated by all chemistries, the second mass spectrum (MS2) is a 'pure' mass spectrum of single precursor ion if there are no isobaric interferences. In other words, MS2 allows one to unambiguously identify a mass peak in MS1. Because the fragmentation for MS2 is achieved in the gas phase, there are also no matrix effects causing reproducibility issues and this means it is now possible to use direct spectrum-to-spectrum matching against a reference library of previously observed MS/MS spectra. Even if there is more than one precursor ion species in the $m / z$-ratio window selected for MS/MS, it will still be relatively easy to deconvolute MS2. In the first ToF-SIMS instrument to demonstrate MS/MS capabilities, collisions of the secondary ions are achieved with argon gas in a field free region before the reflectron but after the exit of the buncher that samples the secondary ion stream, effectively performing high-energy CID [4, 5]. Each precursor and its corresponding product ions continue to travel at the same velocity and are selected using a timed ion gate so the MS/MS configuration can be considered a ToF/ToF. A second manufacturer built an instrument in which a precursor ion can be deflected into a CID cell $[6,7]$. The resulting fragment ions are mass separated in a separate linear TOF with a second pulse counting detector, producing the MS/MS spectrum. A great advantage here is that MS/MS and traditional TOFSIMS data are collected simultaneously from the same analytical area in a single experiment, allowing for MS/MS imaging at a high speed. A third instrument is a so-called Hybrid SIMS, combining a conventional ToF-SIMS instrument with an Orbitrap ${ }^{\mathrm{TM}}$, which allows for MS/MS through higher-energy collisional dissociation (HCD) with a mass resolution of up to 240,000 at $\mathrm{m} / \mathrm{z} 200$ and a mass accuracy below $1 \mathrm{ppm}$ $[8,9]$. This kind of mass resolution and accuracy allows one to determine the chemical formula of each fragment ion in the MS/MS spectrum without any ambiguity.

The following protocol describes how tandem MS can be used to elucidate the structure of unknown or ambiguous mass peaks in biological tissue samples observed during ToF-SIMS imaging. It is largely based 
on a previously published article, where we describe the combination of MALDI-ToF, ToF-SIMS, and Hybrid SIMS to identify markers for pulmonary arterial hypertension in human lung tissue [10]. However, the protocols are modified in order to be applicable to as wide a range of biological tissue samples possible.

2 Materials

2.1 Chemicals and Materials

1. Dry ice.

2. Optimal cutting temperature (OCT) compound.

3. Glass microscope slides and coverslips.

4. Indium tin oxide (ITO) coated glass microscope slides.

5. 4\% paraformaldehyde solution.

6. Hematoxylin solution.

7. $0.5 \%$ Erythrosin B solution.

8. Ethanol.

\subsection{Instrumentation}

1. $-80^{\circ} \mathrm{C}$ freezer.

2. Cryomicrotome.

3. Optical microscope with a digital camera.

4. Freeze-dryer.

5. Vacuum desiccator.

6. Microscope slide scanner.

7. A ToF-SIMS instrument equipped with a Bi LMIG and Ar GCIB that has MS/MS capabilities (See Note 1). 


\section{Methods}

\subsection{Tissue Sectioning}

This is a protocol for the preparation of tissue sections for both ToF-SIMS measurements and complementary histology. The protocol assumes to already have a tissue block of fresh-frozen tissue stored in a $-80^{\circ} \mathrm{C}$ freezer or a liquid nitrogen tank (see Note 2). The authors have applied this protocol to various tissue types of different organisms with little or no variation.

1. Remove frozen tissue block from storage and put the tissue block on dry ice to transport it to the cryotome.

2. Apply OCT to the back of the tissue block to hold it in place during sectioning (see Note 3).

3. Section tissue at $-20^{\circ} \mathrm{C}$ using a cryostat system. The section thickness tends to be limited by the cryostat system and is usually 5-15 $\mu \mathrm{m}$ (see Note 4).

4. For each tissue sample, obtain three serial sections. Thaw-mount sections 1 and 3 on ITO-coated glass microscope slides (see Note 5). Thaw-mount section 2 (middle of the series) on a glass microscope slide.

5. After thaw-mounting, immediately put the slide in a microscope slide box cooled on dry ice.

6. Store the microscope slide box in a $-80^{\circ} \mathrm{C}$ freezer.

\subsection{Tissue Staining}

This is a protocol for the histological staining of the adjacent tissue section on the glass microscope slide. This allows a pathologist to easily identify areas of interest for the ToF-SIMS measurements. This specific protocol was optimized for staining human lung tissue sections.

1. Defrost tissue sections that were thaw-mounted on glass microscope slides as described in 3.1.

2. Fix the sections chemically in $4 \%$ paraformaldehyde solution at room temperature (10 $\mathrm{min})$. 
3. Rinse in tap water (30 s).

4. Stain in hematoxylin solution (1 min).

5. Rinse in tap water (30 s).

6. Counterstain in $0.5 \%$ erythrosin solution ( $3 \mathrm{~s})$.

7. Rinse in tap water (30 sec).

8. Rinse in $70 \%$ ethanol ( $1 \mathrm{~min})$.

9. Rinse in pure ethanol (1 $\mathrm{min})$.

10. Air-dry the tissue.

11. Mount a cover slip and seal with nail polish.

12. Take pictures of the sample using an optical microscope with a digital camera.

\subsection{Sample Preparation Prior to ToF-SIMS}

ToF-SIMS requires ultra-high vacuum (UHV) so the high water content of cells and tissues complicates the sample preparation of biological materials for ToF-SIMS imaging. This part describes the preparation of freeze-dried tissue sections from frozen tissue sections for ToF-SIMS imaging (Note 6). The authors have applied this protocol to various tissue types of different organisms with little or no variation.

1. Remove frozen tissue sections that were thaw-mounted on the ITO-coated glass microscope slides as described in 3.1 from storage and put them on dry ice.

2. Freeze-dry samples using a tray freeze-dryer (see Note 7). If no freeze-dryer is available, dehydrate the tissue sections in a vacuum desiccator for at least $30 \mathrm{~min}$.

3. Scan the ITO coated glass microscope slides with a microscope slide scanner. Alternatively, use an optical microscope with a digital camera.

4. Store the dehydrated tissue sections in a vacuum desiccator prior to SIMS imaging. 


\subsection{ToF-SIMS Imaging}

After the stained images and the images of the corresponding freeze-dried tissue sections are compared, regions of interest can be identified for ToF-SIMS imaging. ToF-SIMS imaging is performed using the Bi liquid metal ion gun (LMIG) as a primary ion beam in order to achieve a high spatial resolution ( $\leq 1 \mu \mathrm{m}$ pixel size).

1. Mount section 1 and introduce the sample holder in the vacuum chamber of the mass spectrometer. The image recorded by the slide scanner can be registered in the ToF-SIMS software's navigator in order to easily find small histological structures.

2. Optimize the $25 \mathrm{keV}$ bismuth LMIG for molecular imaging using steps 3-9.

3. Select $\mathrm{Bi}_{3}{ }^{+}$clusters and operate the gun the high-current bunched mode, which allows for higher mass resolution and has a beam spot of $1-2 \mu \mathrm{m}$.

4. Measure the pulsed primary ion current for $\mathrm{Bi}_{3}{ }^{+}$(about $0.4 \mathrm{pA}$ ) using the Faraday cup provided on each sample holder and a cycle time of $100 \mu$ s (see Note 8).

5. Using a so-called A-Grid, a metallic tuning grid where the A-letter is drawn, the primary ion beam is aligned with the ToF-SIMS camera image.

6. Move the stage to the sample and optimize the distance (or Z-height) between the sample and the extraction cone.

7. Charging of the sample is compensated with the low-energetic (about $20 \mathrm{eV}$ ) electrons of the flood gun. Optimize the charge compensation on the sample surface.

8. Iteratively adjust beam alignment, Z-height and charge compensation on the sample surface for optimal imaging performance.

9. Save all parameters before starting an imaging experiment.

10. Internally calibrate the mass spectra using known mass peaks (see Note 9). 
11. Image the area of interest. Areas larger than $500 \times 500 \mu \mathrm{m}^{2}$ can be imaged by moving the sample stage and image stitching $500 \times 500 \mu \mathrm{m}^{2}$ patches or smaller together.

\subsection{Hybrid SIMS MS/MS}

After analyzing the ToF-SIMS ion image data acquired in 3.3 using the commercial software, unknown or ambiguous mass peaks of interest can be selected for tandem MS. For tandem MS, the Hybrid SIMS'S Thermo Scientific Q Exactive HF Orbitrap is used in this method chapter in combination with the Ar gas cluster ion beam (GCIB) as a quasi-continuous primary ion beam. A typical Hybrid SIMS MS/MS spectrum of a mass peak of interest detected in human lung tissue is provided in Figure 1.

1. Mount tissue section 3 and introduce the sample holder in the vacuum chamber of the mass spectrometer. The image recorded by the slide scanner can be registered in the software's navigator in order to easily locate the same areas analyzed on tissue section 1 in 3.3.

2. Set up the Ar GCIB for tandem MS using 5-20keV $\operatorname{Ar}_{n}$ clusters with $n=1500-3500$ and a spot size of roughly $3 \mu \mathrm{m}$.

3. Measure the primary ion current (about $12 \mathrm{pA}$ at $15 \%$ duty cycle) using the Faraday cup on the sample holder.

4. The Orbitrap analyzer should be calibrated using silver cluster ion patterns from a silver foil standard.

5. Move the stage to the sample areas of interest and acquire spectra with the same field of view used during ToF-SIMS imaging (see Note 10).

6. Record the MS/MS spectrum of the ion of interest using a mass window of $0.4 \mathrm{~m} / \mathrm{z}$ and a collision energy of $40-60 \mathrm{eV}$. 
Notes:

1. In this protocol, the ToF-SIMS imaging is performed using a TOF-SIMS IV instrument (IONTOF GmbH, Münster, Germany). For the MS/MS analyses, the Hybrid SIMS is used (IONTOF GmbH, Münster, Germany), which is a combination of a typical ToF-SIMS instrument with an orbital trapping mass spectrometer (Q Exactive HF, ThermoFisher Scientific GmbH, Bremen, Germany). ToF-SIMS ion images are recorded and analyzed using the SurfaceLab 7 (IONTOF) software.

2. Since the goal is to detect and localize chemical compounds contained in the tissue, care should be taken not to alter its chemical composition during the sample preparation. Consequently, the use of fresh-frozen tissue is recommended as well as avoiding the introduction of any new chemistry through for example chemical fixation, sucrose infiltration, etc. Fresh-frozen means the tissue block should be excised and snap-frozen within 30 to 60 minutes. A small piece of tissue can be flash frozen in a cryogenic liquid such as ethane or isopentane. Otherwise, liquid nitrogen is used.

3. When the tissue block is small, it is possible to embed the sample using a cryomold and an appropriate embedding material to help preserve its shape and make it easier to manipulate the sections. Carboxymethyl cellulose (CMC) and gelatin compounds are more suitable than OCT, because OCT produces a strong signal in positive polarity that obscures lipid peaks and also contains a large amount of salts which could lead to ion suppression. When OCT is used to hold the block in place, care must be taken to avoid cross contamination.

4. The thickness is not really of importance to the ToF-SIMS measurement itself, because ToF-SIMS is a surface analysis technique with an information depth of c. $1 \mathrm{~nm}$ and only c. $20 \mathrm{~nm}$ of subsurface damage. However, one wants to slice as thin as possible in order to ensure the adjacent sections used for replicates or histology are similar. 
5. The tissue sections are thaw-mounted on conductive ITO coated glass microscope slides, because dielectric substrates can negatively affect the ion extraction in a typical ToF-SIMS instrument.

6. Most biological sample preparation techniques for ToF-SIMS involve dehydration of the sample, because a dry sample can be kept at room temperature, allowing convenient sample handling and the use of complementary techniques such as optical microscopy or atomic force microscopy prior to ToF-SIMS analysis. Alternatively, the sample can be imaged in a frozen-hydrated state by performing the ToF-SIMS measurement with a cryogenically cooled stage at a temperature $<-120$ ${ }^{\circ} \mathrm{C}$ below which recrystallization does not occur [11]. This is referred to as cryo-ToF-SIMS and is conceptually analogous to cryo-EM.

7. When freeze-drying, it is imperative that the samples do not thaw before the pressure drops below that of the triple point. If the freeze-dryer has no temperature control, put in a steel plate that has been cooled in the $-80^{\circ} \mathrm{C}$ freezer and place the microscope slides on top of it.

8. This value allows one to calculate the primary ion dose density or 'fluence' before each image acquisition and keep it below the static limit of SIMS $\left(\leq 10^{13}\right.$ ions $\left.\cdot \mathrm{cm}^{-2}\right)$.

9. We refer the reader to reference [12] for a recommended procedure of ToF-SIMS mass calibration.

10. A $500 \times 500 \mu \mathrm{m}^{2}$ area can for example be rastered using $25 \times 25$ pixels with a $20 \mu \mathrm{m}$ spot size. The primary ion dose density is typically in the order of $1 \times 10^{15}$ primary ions per $\mathrm{cm}^{2}$. 
References

1. Vickerman JC, Briggs D (2013) ToF-SIMS: Materials Analysis by Mass Spectrometry. SurfaceSpectra

2. Fearn S (2015) An Introduction to Time-of-Flight Secondary lon Mass Spectrometry (ToF-SIMS) and its Application to Materials Science

3. Tuck M, Blanc L, Touti R, Patterson NH, Van Nuffel S, Villette S, Taveau J-C, Römpp A, Brunelle A, Lecomte S, Desbenoit N (2021) Multimodal Imaging Based on Vibrational Spectroscopies and Mass Spectrometry Imaging Applied to Biological Tissue: A Multiscale and Multiomics Review. Anal Chem 93:445-477 . https://doi.org/10.1021/acs.analchem.0c04595

4. Fletcher JS, Kotze HL, Armitage EG, Lockyer NP, Vickerman JC (2013) Evaluating the challenges associated with time-of-fight secondary ion mass spectrometry for metabolomics using pure and mixed metabolites. Metabolomics 9:535-544 . https://doi.org/10.1007/s11306-012-0487-4

5. Wehrli PM, Lindberg E, Angerer TB, Wold AE, Gottfries J, Fletcher JS (2014) Maximising the potential for bacterial phenotyping using time-of-flight secondary ion mass spectrometry with multivariate analysis and Tandem Mass Spectrometry. Surf Interface Anal 46:173-176 . https://doi.org/10.1002/sia.5505

6. Fisher GL, Hammond JS, Larson PE, Bryan SR, Heeren RMA (2016) Parallel imaging MS/MS TOFSIMS instrument. J Vac Sci Technol B 34:03H126 . https://doi.org/10.1116/1.4943568

7. Fisher GL, Bruinen AL, Ogrinc Potočnik N, Hammond JS, Bryan SR, Larson PE, Heeren RMA (2016) A New Method and Mass Spectrometer Design for TOF-SIMS Parallel Imaging MS/MS. Anal Chem 88:6433-6440 . https://doi.org/10.1021/acs.analchem.6b01022

8. Pirkl A, Moellers R, Arlinghaus H, Kollmer F, Niehuis E, Makarov A, Horning S, Passarelli M, 
Havelund R, Rakowska P, Race A, Shard AG, West A, Marshall P, Newman CF, Alexander M, Dollery C, Gilmore IS (2016) A Novel Hybrid Dual Analyzer SIMS Instrument for Improved Surface and 3D-Analysis. Microsc Microanal 22:340-341 . https://doi.org/DOI:

$10.1017 / \mathrm{S} 1431927616002555$

9. Passarelli MK, Pirkl A, Moellers R, Grinfeld D, Kollmer F, Havelund R, Newman CF, Marshall PS, Arlinghaus H, Alexander MR, West A, Horning S, Niehuis E, Makarov A, Dollery CT, Gilmore IS (2017) The 3D OrbiSIMS-label-free metabolic imaging with subcellular lateral resolution and high mass-resolving power. Nat Methods 14:1175-1183 . https://doi.org/10.1038/nmeth.4504

10. Van Nuffel S, Quatredeniers M, Pirkl A, Zakel J, Le Caer J-P, Elie N, Vanbellingen QP, Dumas SJ, Nakhleh MK, Ghigna M-R, Fadel E, Humbert M, Chaurand P, Touboul D, Cohen-Kaminsky S, Brunelle A (2020) Multimodal Imaging Mass Spectrometry to Identify Markers of Pulmonary Arterial Hypertension in Human Lung Tissue Using MALDI-ToF, ToF-SIMS, and Hybrid SIMS. Anal Chem 53:19 . https://doi.org/10.1021/acs.analchem.0c02815

11. Linner JG, Livesey SA, Harrison DS, Steiner AL (1986) A new technique for removal of amorphous phase tissue water without ice crystal damage: a preparative method for ultrastructural analysis and immunoelectron microscopy. J Histochem Cytochem 34:1123-1135 . https://doi.org/10.1177/34.9.2426340

12. Green FM, Gilmore IS, Seah MP (2006) TOF-SIMS: Accurate mass scale calibration. J Am Soc Mass Spectrom 17:514-523 . https://doi.org/10.1021/jasms.8b02645 
Figure caption:

Figure 1. The negative polarity MS/MS fragment ion spectrum (collision energy of $56.7 \mathrm{eV}$ ) of the $\mathrm{m} / \mathrm{z}$ 885.551 precursor ion from human arterial tissue recorded using the IONTOF Hybrid SIMS instrument. The MS/MS fragment ion spectrum unambiguously identifies this precursor ion as a PI C18:0/C20:4 species, given the $\mathrm{C}_{6} \mathrm{H}_{8} \mathrm{O}_{7} \mathrm{P}^{-}$ion at $\mathrm{m} / \mathrm{z} 223.001$ and the $\mathrm{C}_{6} \mathrm{H}_{10} \mathrm{O}_{8} \mathrm{P}^{-}$ion at $\mathrm{m} / \mathrm{z} 241.012$, which are characteristic fragments for the inositol phosphate headgroup, and the $\mathrm{C}_{18} \mathrm{H}_{35} \mathrm{O}_{2}{ }^{-}$ion at $\mathrm{m} / \mathrm{z} 283.264$ and the $\mathrm{C}_{20} \mathrm{H}_{31} \mathrm{O}_{2}{ }^{-}$ ion at $m / z 303.233$ corresponding to stearic and arachidonic acid, respectively. This mass spectrum also agrees with the commonly occurring product ions for a PI C18:0/C20:4 species published online in LIPIDMAPS, showing spectral matching is now possible. Reproduced from Van Nuffel S. et al. Anal. Chem. 2020, 92:12079-12087 https://pubs.acs.org/doi/10.1021/acs.analchem.0c02815 (reference [10]). Copyright 2020 American Chemical Society. 


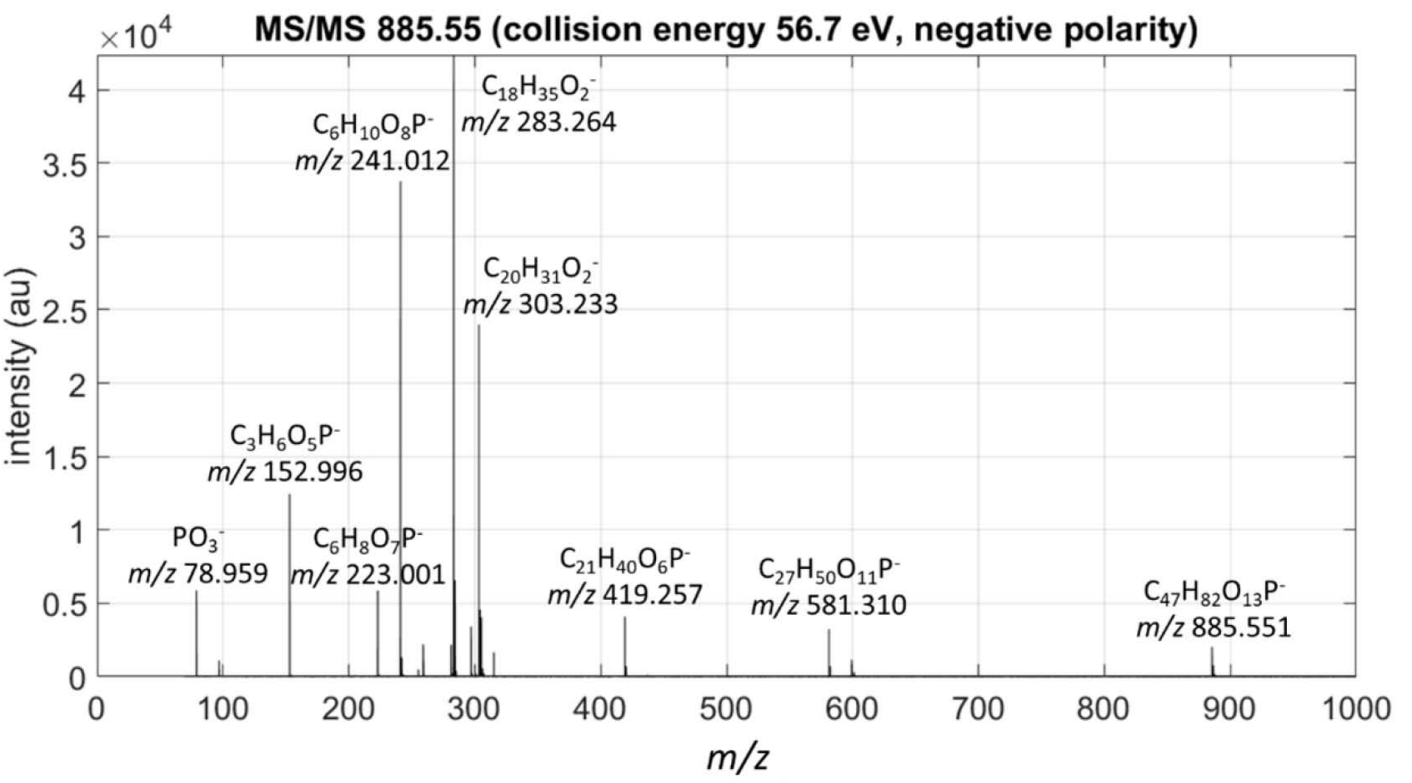

